\title{
Peculiarities of the diffusion-controlled radiation defect accumulation kinetics under high fluencies
}

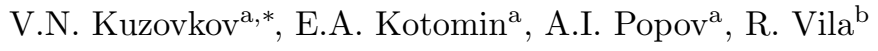 \\ ${ }^{a}$ Institute of Solid State Physics, University of Latvia, Riga, Latvia \\ ${ }^{b}$ CIEMAT, Madrid, Spain
}

\begin{abstract}
Theory is developed for kinetics of the diffusion-controlled radiation defect accumulation in crystalline solids under high fluencies taking into account recently observed correlation between the defect diffusion energy and pre-exponential (known as the Meyer-Neldel rule in chemical kinetics) and their dependence on the radiation fluence (Kotomin et al, J Phys Chem A 122 (2018) 28). The predicted accumulation kinetics could be applied to all kinds of solids. It considerably differs from the commonly used, in particular, suggesting that concentration growth at high fluencies could be nonmonotonous and the saturation defect concentrations independent on the temperature.
\end{abstract}

Keywords: radiation defects; accumulation kinetics; diffusion; fluence effects

\section{Introduction}

As is well studied now, an irradiation of all kinds of solids results in formation of pairs of Frenkel defects - interstitials and vacancies [1, 2, 3, 4. These defects are mobile above certain temperatures and participate in secondary diffusion5 controlled reactions, including complementary defect recombination and aggregation of similar defects leading in oxides and halides to metal colloid or gas bubble formation [5, 6, 7, 8. Since radiation defects considerably affect mechanical and optical properties of materials. Thus prediction and interpretation of the kinetics of defect accumulation upon irradiation is important, especially

10 for optical materials 9, 10, 11, 12, 13, 14, 15, (such as $\mathrm{MgO}, \mathrm{Al}_{2} \mathrm{O}_{3}, \mathrm{MgF}_{2}$ ) used as lenses and windows for nuclear applications.

The simplest model [16, 17] of the process consists of creation of the Frenkel defect pairs $A-B$ with the rate $p$, their diffusion with the diffusion coefficients $D_{A}$ and $D_{B}$, which exponentially depends on the activation energy $E_{c}: D_{c}=$

$15 D_{c}^{0} \exp \left(-E_{c} / k_{B} T\right), c=A, B$. Typically, interstitials are much more mobile than vacancies which are practically immobile in oxides below $1000 \mathrm{~K}[7,8,18,19]$.

\footnotetext{
* Corresponding author

Email address: kuzovkov@latnet.lv (V.N. Kuzovkov )
} 
The main point of this generally accepted model is that the diffusion energy and pre-exponential remain constants independent on the radiation fluencies, which implies that material structure under irradiation remains mostly undistorted.

This model allows us also to include mutual attraction and aggregation of similar defects (e.g. for description of colloid formation [7, 8, 18, 19, 20, 21, 22], where role of defect complexes as sinks for interstitials was in detail discussed). The effective diffusion coefficients of defects bound in these aggregates is considerably decreased. However, defects evaporated from these dynamical aggregates 25 migrate again with their energies $E_{c}$.

\section{Abnormal kinetics}

Our recent analysis of the kinetics of the $\mathrm{F}$ type center (anion vacancies) annealing upon heating due to recombination with mobile complementary interstitial ions in several radiation-resistant ionic solids $\left(\mathrm{MgO}, \mathrm{Al}_{2} \mathrm{O}_{3}, \mathrm{MgF}_{2}\right)$

30 performed in terms of bimolecular reactions have shown unexpected result [19, 23, 24, 25]: the migration energy $E_{a}$ of interstitial and the corresponding preexponential factor $X$ are correlated and considerably depend on the fluence. The parameter $X=N_{0} R D_{0} / \beta$, where $N_{0}$ is initial defect concentration, $R$ recombination radius for dissimilar defects [16, 17, $D_{0}$ - diffusion pre-exponent 35 for interstitials, and $\beta$ heating rate.

Let's start our research with a brief overview of the abnormal behavior of radiation defect annealing kinetics. The work cycle [19, 23, 24, 25] is based on the assumption that recombination of $F$ centers with interstitials is a simple diffusion-controlled recombination of defects with equal initial concentrations.

40 If we use dimensionless concentrations $C(t)$ of defects (they are equal to unity at low temperatures), the law of decreasing concentrations over time is simple:

$$
C(t)=\frac{1}{1+N_{0} \int_{0}^{t} K(t) d t},
$$

where the diffusion-controlled reaction rate $K=4 \pi D R$ is proportional to the mutual diffusion coefficient $D$ [26] and, thus, depends exponentially on the defect migration energy $E_{a}, D=D_{0} \exp \left(-E_{a} / k_{B} T\right)$. Assuming that the temper-

45 ature increase with the heating rate $\beta(t)$, one gets the following relation for the concentration decay

$$
C_{i}=\frac{1}{1+4 \pi\left(N_{0} R D_{0}\right) \int_{T_{0}}^{T_{i}} \exp \left(-E_{a} / k_{B} T\right) \beta^{-1}(T) d T} .
$$

Here we go from a continuous description (kinetics) to a set of temperature values $T_{i}$ used in a specific experiment and corresponding concentration values $C_{i}$. Usually, the temperature in experiments is a linear function of time, $\beta(t)=$

so $\beta=$ const, and we get two control parameters: the migration energy $E_{a}$, and pre-exponential factor $X=N_{0} R D_{0} / \beta$ mentioned before. Finally, we fitted these two key parameters, $E_{a}$ and $X$ to the available experimental kinetics by means of the least square method. 

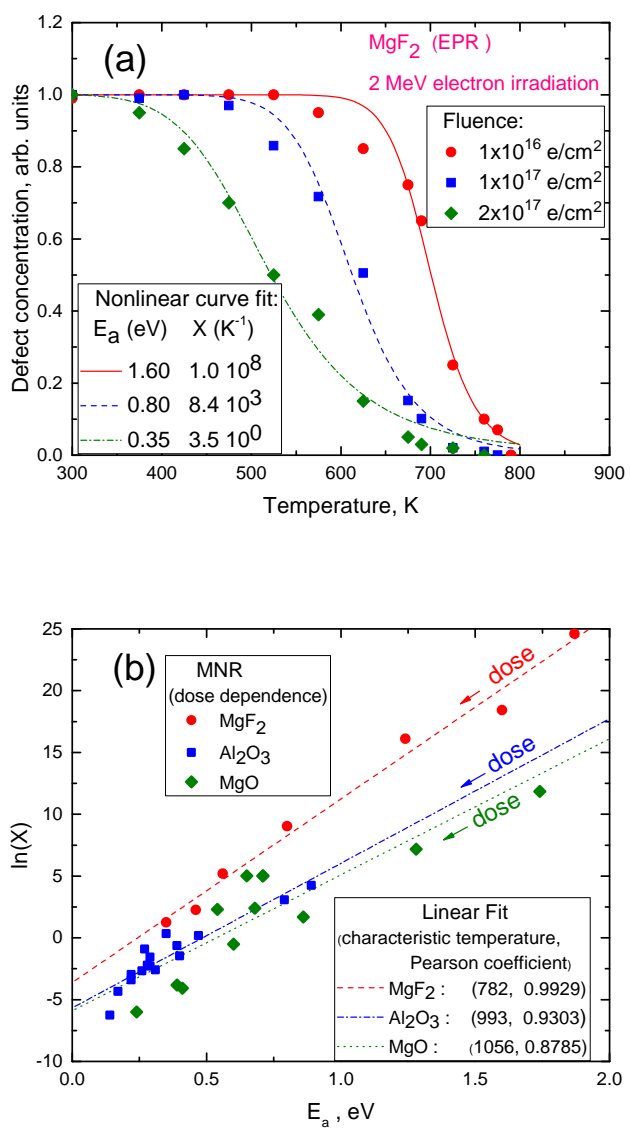

Figure 1: (a) Kinetics of the $F^{+}$center annealing in electron irradiated $\mathrm{MgF}_{2}$ 27 for dierent fluences. (b) Correlation of the effective energies and pre-exponents for $\mathrm{MgF}_{2}$, sapphire, $\mathrm{MgO}$ 23. 
Fig.1a shows fitting of theory to three $\mathrm{F}$ center annealing curves [27] from which we extract parameters $E_{a}, X$ (see the legend in fig.1a) whereas fig.1b shows the result of the analysis for a wide range of experiments, not only for $\mathrm{MgF}_{2}$ but also corundum and $\mathrm{MgO}[23$. As one can see, diffusion energies vary as the function of fluence by a factor of $4-5$, whereas the pre-exponentials by 20 orders of magnitude! Typically, the diffusion energies at small fluencies are close 60 to those theoretically predicted for perfect solid but then dramatically decrease with the fluence, very likely, due to migration in considerably radiation-distorted matrix.

The correlation $E_{a}-X$ (the level of this correlation is characterized by the standard Pearson correlation coeffcient very close to unity, see also the legend in fig.1b) is well described by the relation

$$
\ln (X)=\ln \left(X_{0}\right)+\frac{E_{a}}{k_{B} T_{0}} .
$$

This relation states that the pre-factor $X$ depends on the activation energy, accordingly, which transforms into energy dependent pre-exponent $D_{0}=D_{0}^{*} \exp \left(E_{a} / k_{B} T_{0}\right)$. That is the diffusion coefficient has more complicated as usual dependence on the migration energy [23]

$$
D=D_{0}^{*} \exp \left(\frac{E_{a}}{k_{B} T_{0}}-\frac{E_{a}}{k_{B} T}\right) .
$$

70 This relation was observed for many disordered semiconductors and glasses. There $T_{0}$ is some characteristic temperature (a parameter particular to each material, often associated with the glass transition temperature [28]). The correlation, eq.(3), is known as Meyer-Neldel rule (MNR) [29, 30, 31. The MNR, or compensation law, linearly relates the logarithm of the pre-exponential fac-

75 tors to the activation energy for any processes that are thermally driven in an Arrhenius manner. It was widely observed in many areas of materials science in physics, chemistry, biology and geology [30, 31, although the foundations of this empirical rule are still not fully understood (on the one hand, the theoretical explanation from statistical physics is lacking, but on the other side, the

so MNR can not relate directly to the microscopic details of the kinetic process 28]). The MNR was observed in many thermally activated phenomena such as solid-state diffusion. Surprisingly, in radiation physics, the correlation of kinetic parameters for diffusion has been established only recently 23. This required studying the statistics of a large number of experimental observations (different authors, different materials, different sources of radiation).

As was shown [23, the fluence-dependence of the migration energy could be approximated in the simple way

$$
E_{a}(\delta)=E_{a}(0) \exp \left(-\delta / \delta_{0}\right),
$$

where $E_{a}(0)$ is the migration energy in defectless solid, $\delta_{0}$ is characteristic parameter for material radiation-induced disordering. The parameter $\delta_{0}$ has a 9o simple meaning, it characterizes the radiation resistance of the material. At 
values $\delta \ll \delta_{0}$, the activation energy of the defect diffusion is not much different from that of a defect-free crystal. However at values $\delta>\delta_{0}$ the diffusion of defects in strongly disordered meterials is characterized by small activation barriers and in this sense is similar to the diffusion of atoms in a liquid [32, 33.

\section{Theory and main results}

We want to predict here, how the MNR affects the defect accumulation kinetics under prolonged irradiation. Let us introduce dimensionless parameters: displacements per atom (dpa) $\delta=p R^{3} t$ (the recombination radius $R$ is of the order of the lattice constant), where $p$ is defect concentration produced per unit volume and second (dose rate), $t$ time. Characteristic dose rates in experiment vary from $10^{-7} \mathrm{dpa} / \mathrm{s}$ to $10^{-4} \mathrm{dpa} / \mathrm{s}$. Assuming the irradiation time $24 \mathrm{~h}\left(10^{5} \mathrm{~s}\right)$, we estimate $\delta=10^{-2}-10^{1} \mathrm{dpa}$. Let us take the upper bound in our calculation, $\delta_{\max }=1$ dpa.

It is convenient to introduce the dimesionless parameter

$$
\epsilon=E_{a}(0) / k_{B} T_{0},
$$

105 One can estimate from the data shown in the fig.1b and ref. 23] that $\epsilon=$ 30 for $\mathrm{MgF} 2, \epsilon=10$ in $\mathrm{Al}_{2} \mathrm{O}_{3}$, and $\epsilon=20$ in $\mathrm{MgO}$. Let us fix $\epsilon=20$ in further calculations. We employ the simple rate equation [16, 17] for defect concentrations $n(t)$ increase upon irradiation

$$
\frac{d n}{d t}=p-K n^{2}
$$

where $K=4 \pi D R$ is the Smoluchowski rate of diffusion controlled recombination [26] of vacancies and interstitials. The stead-state solution of eq.(7) in the high temperature limit $\tau=T / T_{0}=1$ reads

$$
n_{0}^{2}=\frac{p}{4 \pi R D_{0}^{*}} .
$$

The $n_{0}$ gives the minimum saturation concentration at any temperature $T<T_{0}$ but fixed parameters $p$ and $D_{0}^{*}$. Let us introduce the dimensionless concentrations $\nu=n R^{3}, \nu_{0}=n_{0} R^{3}$. Their meaning is average defect numbers in the unit cell volume. Now eq. $(7)$ could be rewritten as

$$
\frac{d \nu}{d \delta}=1-\left(\nu / \nu_{0}\right)^{2} f(\tau, \delta),
$$

where

$$
f(\tau, \delta)=\exp \left(-\epsilon \frac{1-\tau}{\tau} e^{-\delta / \delta_{0}}\right),
$$

This equation could be numerically solved. Let us assume hereafter $n_{0}=10^{18}$ def $/ \mathrm{cm}^{3}$ and $\nu_{0}=10^{-3}$ (the choice is not fundamental and is related to the specification of calculations) and analyze how the accumulation kinetics depends 

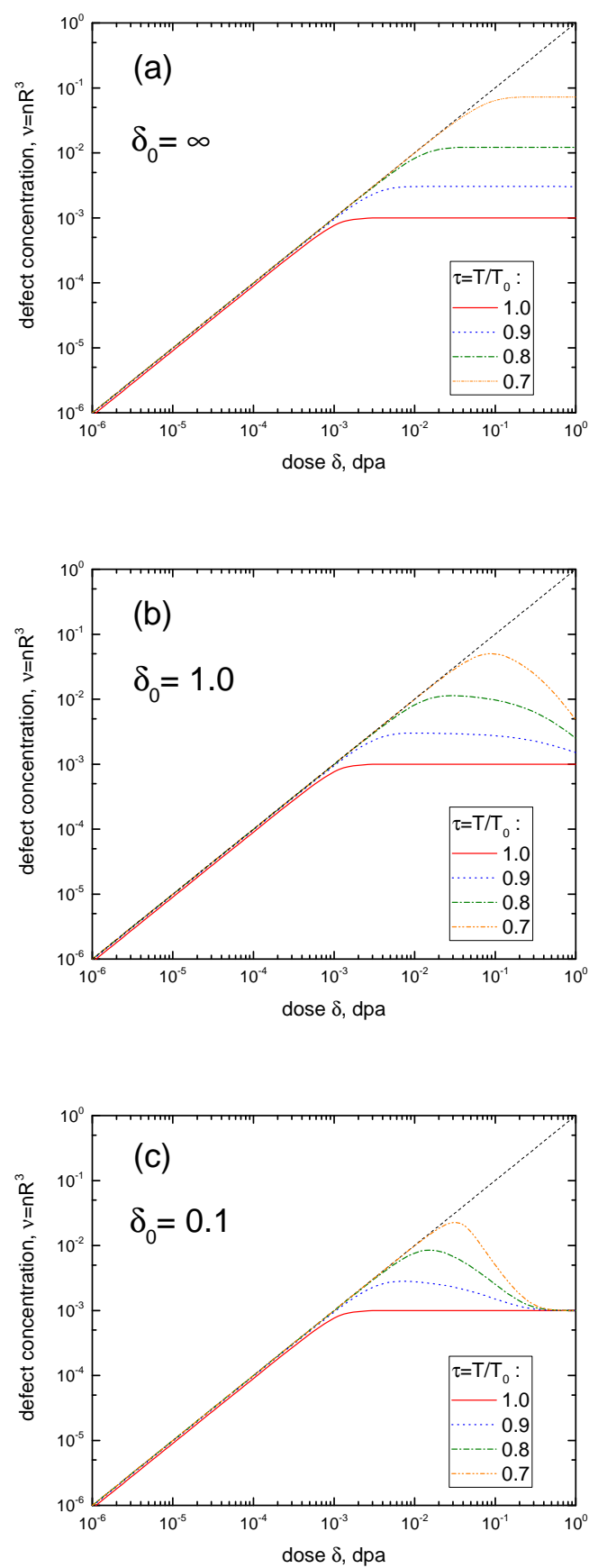

Figure 2: The defect accumulation kinetics with migration energy depending on the fluence. 

$\delta_{0}$. In other words, we estimate the effect of the dose-dependence of migration energy in disordered solids.

Fig.2a shows the defect accumulation kinetics with constant migration energy (no MNR, formally $\delta_{0}=\infty$ ). The saturation concentration is the lower, the

125 higher temperature (and faster diffusion-controlled recombination). The minimum concentration corresponds to $\tau=1$. Incorporation of the MNR in fig. $2 \mathrm{~b}$ shows new features: the kinetics becomes nonmonotonous with fluence and the saturation concentrations are close at all temperatures. In other words, fast defect migration in strongly disordered solids becomes similar to that in liquids 32, 33. This effect is even better pronounced in fig.2b where the saturation concentration no longer depends on the temperature. We predict this effect to be observed, e.g. for prolonged high energy electron irradiation of $\mathrm{MgF}_{2}$ [27. Our calculations demonstrate the important effect of the MNR on the defect accumulation kinetics in strongly irradiated solids. Unfortunately, we did not find sufficient experimental data to apply our theory; the necessary experimental data (e.g. [12, 13, 15]) should show clear saturation and measured at different temperatures.

The accumulation of various color centers was most comprehensively studied in the alkali halides and some simple oxides. In particular, clear effects of 140 temperature, crystal structure, impurities and the type of radiation 34, 35, 36, 37, 38, 39, 40, 41 were reported. Moreover, it has been more than once demonstrated that the saturation effects are most easily observed in case of ion irradiation.

\section{Conclusions}

Summing up, we demonstrated that dependence of the defect diffusion energy and pre-exponential on the radiation fluence is important for understanding and prediction of the defect accumulation kinetics and thus materials radiation stability stability at high fluencies. In particular, the saturation concentration could be independent on the temperature. We have shown here how de-

150 fect annealing kinetics after irradiation with different fluencies could be used for prediction of the defect accumulation kinetics upon prolonged irradiation. Note that in order to predict qualitatively new effects, we used very simple model of the recombination kinetics, neglecting defect clustering and trapping. As was mentioned in Introduction, control of the defect concentrations is of a 5 key importance for prediction of optical properties of materials in harsh radiation environment. Thus, our study could be useful for a wide class of optical materials, including nitrides, carbides and oxides, both crystals and ceramics (refs. 42, 43, 44, 45]).

\section{Acknowledgements}

160

We are grateful to A. Lushchik and E. Shablonin for numerous and valuable discussions. This work has been carried out within the framework of the 
EUROfusion Consortium and has received funding from the Euratom research and training programme 2014-2018 and 2019-2020 under grant agreement No 633053. The views and opinions expressed herein do not necessarily reflect those of the European Commission.

The raw/processed data required to reproduce these findings cannot be shared at this time as the data also forms part of an ongoing study.

\section{References}

[1] S.J. Zinkle, C. Kinoshita, J. Nucl. Mater. 251 (1997) 200-217.

$170[2]$ K. Nordlund, S.J. Zinkle, A.E. Sand, F. Granberg, R.S. Averback, R.E. Stoller, T. Suzudo, L. Malerba, F. Banhart, W.J. Weber, F. Willaime, S.L. Dudarev, D. Simeone, J. Nucl. Mater. 512 (2018) 450-479.

[3] G.S. Was, D. Petti, S. Ukai, S. Zinkle, J. Nucl. Mater. 527 (2019) 151837.

[4] B.P. Uberuaga, S. Choudhury, and A. Caro, J. Nucl. Mater. 462 (2015) 402-408.

[5] J.M. Bunch, J.G. Hoffman, A.H. Zeltmann, J.Nucl.Mat. 73 (1978) 65-69.

[6] G.P. Pells, J. Am.Cer.Soc. 77 (1994) 368-377.

[7] V.N. Kuzovkov, A.I. Popov, E.A. Kotomin, M.A. Monge, R. Gonzalez, and Y. Chen, Phys. Rev. B 64 (2001) 064102 (p. 1-5).

[8] E.A. Kotomin, V.N. Kuzovkov, A.I. Popov, M.A. Monge, R. Gonzalez, and Y. Chen, Nucl. Instr. Meth. Phys. Research B 191 (2002) 208-211.

[9] F. Mota, C.J. Ortiz, R. Vila, N. Casal, A. Garcia, A. Ibarra, J. Nucl. Mater. 422 (Suppl.1) (2013) 5699-5704.

[10] R. DeSalvo, A. Said, D.J. Hagan, E.W. Van Stryland, M. Sheik-Bahae, IEEE. J. Quantum Electr. 32 (1996) 1324-1333.

[11] T. Shikama, K. Yasuda, S. Yamamoto, C. Kinoshita, S.J. Zinkle, E.R. Hodgson, J. Nucl. Mater. 271-272 (1999) 560-568.

[12] B.D. Evans, J. Comas, P.R. Malmberg, Phys. Rev. B 6 (1972) 2453-2462.

[13] B.D. Evans, Phys. Rev. B 9 (1974) 5222-5235.

[14] J.T. Graham, Y. Zhang, W.J. Weber, J. Nucl. Mater. 498 (2018) 400-408.

[15] B. Henderson, R.D. King, Philosophical Magazine, 13 (1966) 1149-1156.

[16] E.A. Kotomin and V.N. Kuzovkov, Rep. Prog. Phys. 55 (1992) 2079-2188.

[17] E.A. Kotomin and V.N. Kuzovkov, Modern Aspects of Diffusion-Controlled Processes: Cooperative Phenomena in Bimolecular Reactions, Amsterdam, Elsevier (Vol. 34 in a series of Comprehensive Chemical Kinetics), 1996. 
[18] E.A. Kotomin, V.N. Kuzovkov, and A.I. Popov, Radiat. Def. Eff. Solids, 155 (2001) 113-125.

[19] E.A. Kotomin, V.N. Kuzovkov, A.I. Popov, and R. Vila, Nucl. Instr. Meth. Phys. Res. B 374 (2016) 107-110.

200

[32] Y.I. Frenkel, Kinetic Theory of Liquids, Oxford, Clarendon Press, 1946.

[33] T.E. Faber, An Introduction to the Theory of Liquid Metals, Cambridge, Cambridge University Press, 1972.

[34] G. Baubekova, A. Akilbekov, A.I. Popov, E. Shablonin, E. Vasilchenko, M. Zdorovets, A. Lushchik, Radiat. Meas., 135 (2020) 106379 (p. 1-6).

[35] D.V. Ananchenko, S.V. Nikiforov, V.N. Kuzovkov, A.I. Popov, G.R. Ramazanova, R.I. Batalov, R.M. Bayazitov, H.A. Novikov, Nucl. Instr. Meth. Phys. Res. B, 466 (2020) 1-7. 
[36] G. Baubekova, A. Akilbekov, E. Feldbach, R. Grants, I. Manika, A.I.

[45] M. V. Zdorovets, A. L. Kozlovskiy, Surface and Coatings Technology, 383

(2020) 125286 (p.1-6). Popov, K. Schwartz, E. Vasil'chenko, M. Zdorovets, A. Lushchik, Nucl. Instr. Meth. Phys. Res. B, 463 (2020) 50-54.

[37] G. Baubekova, A. Akilbekov, E.A. Kotomin, V.N. Kuzovkov, A.I. Popov, E. Shablonin, E. Vasil'chenko, M. Zdorovets, A. Lushchik, Nucl. Instr. Meth. Phys. Res. B, 462 (2020) 163-168.

[38] A.I.Popov, E.Balanzat, Nucl. Instr. Meth. Phys. Res. B, 166-167 (2000) 545-549.

[39] A. Akilbekov, T. Nurakhmetov, A. Elango, Phys. Stat. Sol. B, 100 (1980) 289-296.

[40] M.V. Sorokin, K. Schwartz, C. Trautmann, A. Dauletbekova, A.S. El-Saide, Nucl. Instr. Meth. Phys. Res. B, 326 (2014) 307-310

[41] K.Schwartz, M.V.Sorokin, A.Lushchik, Ch.Lushchik, E.Vasilchenko, R.M.Papaleo, D. de Souza, A.E.Volkov, K.-O.Voss, R.Neumann, C.Trautmann, Nucl. Instr. Meth. Phys. Res. B, 266 (2008) 2736-2740.

[42] A. Kozlovskiy, K. Dukenbayev, I. Kenzhina, D. Tosi, M. Zdorovets, Vacuum, 155 (2018) 412-422.

[43] T. Gladkikh, A. Kozlovskiy, I. Kenzhina, K. Dukenbayev, M. Zdorovets, Vacuum, 161 (2019) 103-110.

[44] A. V. Trukhanov, A. L. Kozlovskiy, A. E. Ryskulov, V. V. Uglov, S. B. Kislitsin, M. V. Zdorovets, S. V. Trukhanov, T. I. Zubar, K. A. Astapovich, D. I. Tishkevich, Ceramics International, 45 (2019) 15412-15416.

Institute of Solid State Physics, University of Latvia as the Center of Excellence has received funding from the European Union's Horizon 2020 Framework Programme H2020-WIDESPREAD-01-2016-2017-TeamingPhase2 under grant agreement No. 739508, project CAM ART ${ }^{2}$ 